\title{
Inteligencia artificial en la detección de pólipos colónicos: qué dicen los estudios
}

\section{Artificial intelligence in the detection of colonic polyps: what the studies say}

Ricardo Cepeda-Vásquez, MD. ${ }^{*}$

\author{
GaCCESO ABierTo \\ Citación: \\ Cepeda-Vásquez R. Inteligencia artificial \\ en la detección de pólipos colónicos: \\ qué dicen los estudios. Rev Colomb \\ Gastroenterol. 2021;36(1):2-6. https://doi. \\ org/10.22516/25007440.726
}

Médico especialista en medicina interna, Universidad Javeriana, especialista en gastroenterología, Universidad Militar. Gastroenterólogo, Organización Keralty. Bogotá, Colombia.

*Correspondencia: Ricardo Cepeda-Vásquez. doctorcepeda@gmail.com

Fecha recibido: $12 / 02 / 21$ Fecha aceptado: $15 / 02 / 21$
Es de conocimiento general el impacto que ha tenido la colonoscopia y la polipectomía de pólipos adenomatosos en la reducción de la incidencia y la mortalidad del cáncer colorrectal (CCR); sin embargo, parece que los avances endoscópicos no se han traducido en una reducción de las tasas de cáncer de intervalo $(1,2)$.

La tasa de detección de adenomas (TDA) es un indicador de calidad conocido durante la realización de colonoscopia y es definido como la proporción de pacientes a los que se les detecta uno o más adenomas durante el procedimiento, y se considera aceptable una tasa global de detección mayor del $25 \%$ ( $\geq 30 \%$ en varones y $\geq 20 \%$ en mujeres) durante las colonoscopias de tamizaje (3). Este indicador permite evaluar la eficacia de un endoscopista para detectar lesiones adenomatosas y es de suma importancia, ya que por cada aumento del $1 \%$ en la TDA del examinador se presentará una disminución del $3 \%$ de CCR (4). Infortunadamente, se ha demostrado en diversos estudios que la TDA presenta una gran variabilidad entre los endoscopistas con rangos entre un $7 \%$ y $53 \%$ (4), con una pérdida del $20 \%$ al $30 \%$ de los adenomas durante las colonoscopias de detección (5), convirtiéndose en el origen del CCR de intervalo hasta en el $9 \%$ de todos los casos (6).

Los factores causales son múltiples: unos inherentes al paciente con preparaciones inadecuadas o lesiones ubicadas en zonas de difícil evaluación; otros derivados del endoscopista, cuya variabilidad interoperador puede estar relacionada con falta de experiencia o errores de reconocimiento, realización de procedimientos con indicadores de calidad subóptimos y otros factores contributivos como la falta de motivación, la distracción o el deterioro del estado de alerta (7). Por tanto, las estrategias para minimizar las lesiones no detectadas durante la colonoscopia son de gran importancia, pasando por la capacitación médica a endoscopistas y enfermeros, la realización de colonoscopias de alta calidad, el uso de equipos de alta definición, tecnologías de imágenes avanzadas como la de banda estrecha (NBI) o de LCI (linked color imaging), al igual que dispositivos auxiliares como cap o el endocuff, que en algunos estudios evidencian mejoría en la tasa de detección de pólipos y adenomas (8-12).

En el mismo sentido, el hecho de que buena parte del diagnóstico de las afecciones gastrointestinales se apoya en estudios endoscópicos con imágenes digitales, convierte al diagnóstico asistido por computadora (CAD) y a la inteligencia artificial (IA) en herramientas de interés, que pueden reducir los errores no intencionados en la práctica de la colonoscopia, como la detección de pólipos (CADe) y la caracterización diagnóstica de los mismos (CADx) (13). La primera, CADe, tiene el potencial de disminuir la tasa de falla en el reconocimiento de pólipos, lo que contribuye a mejorar la detec- 
ción de adenomas; mientras que CADx está enfocada en mejorar la precisión del diagnóstico óptico de pólipos colorrectales, lo que se podría traducir en una reducción en el número de polipectomías innecesarias, de lesiones distales no neoplásicas y eventualmente en la implementación del proceso de resección y descarte de las mismas (13).

Estos sistemas de IA se han desarrollado sobre un modelo de aprendizaje automático y supervisado que extrae las covariables de los datos de entrenamiento a partir de imágenes y logra el reconocimiento o la clasificación de patrones para posteriormente utilizarlos en la predicción de resultados (14). Entre varios modelos existentes, el modo de aprendizaje profundo (DL), basado en redes neuronales convolucionales $(\mathrm{CNN})$, ha demostrado un entendimiento sobresaliente en el análisis de estas imágenes. Estas redes están conformadas por múltiples capas con neuronas artificiales, que actúan como un filtro para la extracción de las características importantes de la imagen (14). Se cuenta con capas de conexión que pueden agilizar el cálculo subyacente y, una vez conectadas todas las capas, combinar las características de la imagen, para crear un modelo que permite clasificar diferentes resultados con el menor grado de error (15) sin necesidad de intervención o indicación humana.

Los estudios iniciales se realizaron de forma exploratoria o retrospectiva utilizando imágenes almacenadas y probablemente sobreestimaron los resultados. En el estudio de Urban se diseñó y entrenó una CNN para la detección de pólipos, basados en una colección de 20 vídeos con una duración de 5 horas, con una precisión diagnóstica del $96,4 \%$ y un área bajo la curva (ROC) de 0,991. Se observó que el número total de pólipos detectados por la IA era significativamente mayor (en un $37 \%$ ), comparado con lo reportado por endoscopistas expertos, aunque la diferencia estaba dada por lesiones pequeñas de 1-6 $\mathrm{mm}$ de tamaño (16). Wang realizó otro estudio prospectivo con más de 1000 pacientes (17) y los aleatorizó a colonoscopia con o sin asistencia de IA, cuyo objetivo primario era la TDA, y se documentó un incremento en la tasa del 20,3 \% al 29,1\% y la media de número de adenomas por paciente aumentó de 0,31 a 0,53 . Tuvo en común con el estudio anterior, que el incremento era a expensas de pólipos diminutos, sin obtenerse una diferencia estadística en la detección de adenomas avanzados.

Gómez y colaboradores en el artículo "Detección automática de pólipos colorrectales con técnicas de inteligencia artificial”, publicado en este número de la Revista Colombiana de Gastroenterología, ilustran conceptual y ampliamente sobre el desarrollo de la arquitectura de una $\mathrm{CNN}$ de aprendizaje profundo. Al tomar $3 \mathrm{CNN}$ validadas como son InceptiónV3, Vgg16 y ResNet50, y a través de datos recopilados de 6 bases de imágenes con 1875 casos de pólipos colorrectales lograron obtener un índice de exactitud diagnóstica para la detección de pólipos del 0,7 con una sensibilidad del 0,89 , especificidad de 0,71 y una ROC de 0,87 . Se observó una mejor sensibilidad de la IA, al compararse contra endoscopistas experimentados, y se planteó la posibilidad de ser usados como segundos lectores en un servicio de colonoscopia, que podría subsanar los errores humanos de una colonoscopia en tiempo real. A su vez, resaltan su papel en la reducción de la brecha entre el experto y el examinador en entrenamiento, tanto en la tasa de detección como en la caracterización de adenomas.

Aunque los diseños y algoritmos de estos estudios no son uniformes, la IA ha demostrado la capacidad que le permite la diferenciación histológica, demostrando mejores resultados en aquellos estudios que utilizaron un modelo de aprendizaje profundo, como lo demuestra el grupo de Byrne, quienes con imágenes endoscópicas de video de pólipos colorrectales lograron diferenciar entre un pólipo hiperplásico y uno adenomatoso, con una sensibilidad del $98 \%$ y una especificidad del $83 \%$ (18). En el estudio del Chen se utilizó colonoscopia de aumento y NBI y se reprodujo una sensibilidad del $96 \%$ con una especificidad del $78,2 \%$ en la diferenciación histológica (19).

En los últimos años se han publicado varios estudios prospectivos, evaluando el papel del sistema CADe con aprendizaje profundo basado en $\mathrm{CNN}$, con alertas visuales que permiten identificar lesiones polipoides en el monitor de endoscopia en tiempo real. En el estudio de Wang en 2020 se evaluaron 369 pacientes asignados alternadamente a colonoscopia CADe y colonoscopia convencional, y se observó que la tasa de pérdida de adenoma fue significativamente menor en el $13,89 \%$ con la IA frente al $40 \%$ con la colonoscopia de rutina. Igualmente, la tasa de pérdida de pólipos fue significativamente menor en el $12,98 \%$ frente al $45,90 \%$, respectivamente (20). Repici en el mismo año realizó un ensayo controlado aleatorizado (ECA) multicéntrico para evaluar la seguridad y eficacia de un sistema CADe en tiempo real, y observó que la TDA fue significativamente mayor en el grupo CADe, en un $54,8 \%$, frente a la colonoscopia convencional, en el 40,4\%. Los adenomas de $5 \mathrm{~mm}$ o menos se detectaron en el grupo CADe en un 33,7 \% frente al 26,5\% del grupo de control y se observó una situación similar con los adenomas de 6 a $9 \mathrm{~mm}$, en el 10,6 \% en el grupo CADe frente al 5,8\% en el grupo de control (21).

Gong evaluó a 704 pacientes con un sistema de CNN profundas (ENDOANGEL) para monitorizar la TDA, la cual fue significativamente mayor en el grupo de IA en un $16 \%$ frente al $8 \%$ del grupo de control (22). Otro metaanálisis de 5 ECA con 4354 pacientes evidenció que la TDA combinada fue significativamente mayor en el grupo de CADe (36,6 \%) que en el grupo de control $(25,2 \%)$ (23). En la revisión sistemática de Aziz y Cruda con 2815 pacientes de $3 \mathrm{ECA}$, la colonoscopia con IA resultó en una 
mejoría significativa del $32,9 \%$ frente a la TDA de $20,8 \%$ del grupo de control, con una tasa de detección de pólipos (TDP) del 43,0 \% frente al 27,8\%, respectivamente (24).

El mayor metaanálisis de predicción histológica de pólipos fue recientemente publicado por Lui y colaboradores, en el que se analizaron 18 estudios con un total de 7680 imágenes de pólipos colónicos, con una sensibilidad combinada en la predicción histológica del 92,3 \% y una especificidad del 89,8 \%, con un área bajo la curva (AUC) de la IA de 0,96 (25). El AUC de la IA combinado con NBI fue significativamente mayor que sin NBI $(0,98$ frente a 0,$84 ; p<0,01)$. En el mismo estudio, durante el análisis de caracterización de pólipos diminutos utilizando un modelo DL con NBI sin magnificación, el valor predictivo negativo combinado fue del 95,1 \%. En el caso de los pólipos no adenomatosos, la sensibilidad para diferenciar entre uno hiperplásico y un adenoma serrado sésil fue del $95,2 \%$ y la especificidad, del 95,9\%. Sin embargo, las tasas agrupadas de riesgo relativo de TDA avanzada y TDA de lesiones aserradas sésiles fueron comparables entre la colonoscopia asistida por IA y la estándar (25).

Estos hallazgos parecen confirmar la hipótesis de que los sistemas CADe basados en IA durante la colonoscopia en tiempo real mejoran significativamente la TDA y permiten una buena predicción histológica, en particular combinado con NBI.

Ahora bien, la IA no es infalible, pues se ha demostrado que el $20 \%$ de los adenomas omitidos no se detectaron tampoco con la IA. Probablemente, por encontrarse detrás de pliegues o en áreas del colon de difícil evaluación o camuflarse detrás de una mala preparación intestinal. Desde otra perspectiva, implica que el $80 \%$ de las lesiones no detectadas serán visibles en el monitor y el subdiagnóstico sería atribuible al factor humano, lo cual resalta el papel de la IA como un lector adicional para el endoscopista en tiempo real (14).
Dentro de las desventajas de la CADx, en primer lugar, se señala un incremento en el tiempo de la colonoscopia, al pasar de 35 a 47 segundos por pólipo evaluado con CADx. En segundo lugar, el resultado de CADe o CADx podría distraer la concentración del endoscopista, conduciéndolo a la omisión o a una caracterización errónea de los pólipos (14). Un tercer inconveniente puede derivarse de una relación de dependencia de la CADe por parte de la generación de nuevos endoscopistas, que puede interferir con el desarrollo de las habilidades en el reconocimiento y diferenciación de los pólipos colónicos (10), al depositar una confianza excesiva en la herramienta, que, como ya fue mencionado, no es perfecta y exige en forma compensatoria del esmero y las habilidades del endoscopista para mejorar la TDA. Es por esto que el acompañamiento motivacional de un examinador experimentado durante la instrucción de endoscopistas junior puede equilibrar la balanza y convertir la IA en una plataforma de gran valor educativo.

Con la llegada al escenario endoscópico de estas tecnologías, los endoscopistas deben aprender su utilidad y conocer sus ventajas y limitaciones para lograr explotar sus beneficios de la mejor forma posible durante la práctica clínica diaria.

En conclusión, los sistemas de IA tienen un desarrollo exponencial en la endoscopia digestiva y las subcategorías como el aprendizaje profundo y las redes neuronales sin duda aportarán mucho en el diagnóstico de las enfermedades gastrointestinales, de tal modo que estas tecnologías se verán posicionadas como asistentes virtuales durante la colonoscopia en tiempo real y ayudarán a mejorar la TDA y la tasa de pérdida de adenomas, así como a reducir la incidencia del carcinoma colorrectal y, probablemente, disminuir la frecuencia de neoplasias de intervalo.

\section{REFERENCIAS}

1. Patel SG, Ahnen DJ. Prevention of interval colorectal cancers: what every clinician needs to know. Clin Gastroenterol Hepatol. 2014;12(1):7-15. https://doi. org/10.1016/j.cgh.2013.04.027

2. Murthy SK, Benchimol EI, Tinmouth J, James PD, Ducharme R, Rostom A, Dubé C. Temporal trends in postcolonoscopy colorectal cancer rates in 50- to 74-year-old persons: a population-based study. Gastrointest Endosc. 2018;87(5):1324-1334.e4. https://doi.org/10.1016/j. gie.2017.12.027

3. Kaminski MF, Thomas-Gibson S, Bugajski M, Bretthauer M, Rees CJ, Dekker E, Hoff G, Jover R, Suchanek S, Ferlitsch M, Anderson J, Roesch T, Hultcranz R, Racz I, Kuipers EJ, Garborg K, East JE, Rupinski M, Seip B,
Bennett C, Senore C, Minozzi S, Bisschops R, Domagk D, Valori R, Spada C, Hassan C, Dinis-Ribeiro M, Rutter MD. Performance measures for lower gastrointestinal endoscopy: a European Society of Gastrointestinal Endoscopy (ESGE) Quality Improvement Initiative. Endoscopy. 2017;49(4):378-397. https://doi. org/10.1055/s-0043-103411

4. Corley DA, Jensen CD, Marks AR, Zhao WK, Lee JK, Doubeni CA, Zauber AG, de Boer J, Fireman $\mathrm{BH}$, Schottinger JE, Quinn VP, Ghai NR, Levin TR, Quesenberry CP. Adenoma detection rate and risk of colorectal cancer and death. N Engl J Med. 2014;370(14):1298-306. https://doi.org/10.1056/ NEJMoa1309086 
5. Ahn SB, Han DS, Bae JH, Byun TJ, Kim JP, Eun CS. The Miss Rate for Colorectal Adenoma Determined by Quality-Adjusted, Back-to-Back Colonoscopies. Gut Liver. 2012;6(1):64-70. https://doi.org/10.5009/ gnl.2012.6.1.64

6. Sanduleanu S, Masclee AM, Meijer GA. Interval cancers after colonoscopy-insights and recommendations. Nat Rev Gastroenterol Hepatol. 2012;9(9):550-4. https://doi. org/10.1038/nrgastro.2012.136

7. Zhao S, Wang S, Pan P, Xia T, Chang X, Yang X, Guo L, Meng Q, Yang F, Qian W, Xu Z, Wang Y, Wang Z, Gu L, Wang R, Jia F, Yao J, Li Z, Bai Y. Magnitude, Risk Factors, and Factors Associated With Adenoma Miss Rate of Tandem Colonoscopy: A Systematic Review and Metaanalysis. Gastroenterology. 2019;156(6):1661-1674.e11. https://doi.org/10.1053/j.gastro.2019.01.260

8. Kudo T, Saito Y, Ikematsu H, Hotta K, Takeuchi Y, Shimatani M, Kawakami K, Tamai N, Mori Y, Maeda Y, Yamada M, Sakamoto T, Matsuda T, Imai K, Ito S, Hamada K, Fukata N, Inoue T, Tajiri H, Yoshimura K, Ishikawa H, Kudo SE. New-generation full-spectrum endoscopy versus standard forward-viewing colonoscopy: a multicenter, randomized, tandem colonoscopy trial (J-FUSE Study). Gastrointest Endosc. 2018;88(5):854-864. https://doi. org/10.1016/j.gie.2018.06.011

9. Atkinson NSS, Ket S, Bassett P, Aponte D, De Aguiar S, Gupta N, Horimatsu T, Ikematsu H, Inoue T, Kaltenbach T, Leung WK, Matsuda T, Paggi S, Radaelli F, Rastogi A, Rex DK, Sabbagh LC, Saito Y, Sano Y, Saracco GM, Saunders BP, Senore C, Soetikno R, Vemulapalli KC, Jairath V, East JE. Narrow-Band Imaging for Detection of Neoplasia at Colonoscopy: A Meta-analysis of Data From Individual Patients in Randomized Controlled Trials. Gastroenterology. 2019;157(2):462-471. https://doi. org/10.1053/j.gastro.2019.04.014

10. Leung WK, Lo OS, Liu KS, Tong T, But DY, Lam FY, Hsu AS, Wong SY, Seto WK, Hung IF, Law WL. Detection of colorectal adenoma by narrow band imaging (HQ190) vs. high-definition white light colonoscopy: a randomized controlled trial. Am J Gastroenterol. 2014;109(6):855-63. https://doi.org/10.1038/ajg.2014.83

11. Leung WK, Guo CG, Ko MKL, To EWP, Mak LY, Tong TSM, Chen LJ, But DYK, Wong SY, Liu KSH, Tsui V, Lam FYF, Lui TKL, Cheung KS, Lo SH, Hung IFN. Linked color imaging versus narrow-band imaging for colorectal polyp detection: a prospective randomized tandem colonoscopy study. Gastrointest Endosc. 2020;91(1):104-112. e5. https://doi.org/10.1016/j.gie.2019.06.031

12. Yoshida N, Hisabe T, Ikematsu $H$, Ishihara $H$, Terasawa M, Inaba A, Sato D, Cho H, Ego M, Tanaka Y, Yasuda R, Inoue K, Murakami T, Inada Y, Itoh Y, Saito Y. Comparison Between Linked Color Imaging and Blue Laser Imaging for Improving the Visibility of Flat Colorectal Polyps: A Multicenter Pilot Study. Dig Dis Sci. 2020;65(7):20542062. https://doi.org/10.1007/s10620-019-05930-x
13. Vinsard DG, Mori Y, Misawa M, Kudo SE, Rastogi A, Bagci U, Rex DK, Wallace MB. Quality assurance of computeraided detection and diagnosis in colonoscopy. Gastrointest Endosc. 2019;90(1):55-63. https://doi.org/10.1016/j. gie.2019.03.019

14. Lui TKL, Leung WK. Is artificial intelligence the final answer to missed polyps in colonoscopy? World J Gastroenterol. 2020 Sep 21;26(35):5248-5255. https:// doi.org/10.3748/wjg.v26.i35.5248

15. Takiyama H, Ozawa T, Ishihara S, Fujishiro M, Shichijo S, Nomura S, Miura M, Tada T. Automatic anatomical classification of esophagogastroduodenoscopy images using deep convolutional neural networks. Sci Rep. 2018;8(1):7497. https://doi.org/10.1038/s41598-018-25842-6

16. Urban G, Tripathi P, Alkayali T, Mittal M, Jalali F, Karnes W, Baldi P. Deep Learning Localizes and Identifies Polyps in Real Time With 96\% Accuracy in Screening Colonoscopy. Gastroenterology. 2018;155(4):1069-1078. e8. https://doi.org/10.1053/j.gastro.2018.06.037

17. Wang P, Berzin TM, Glissen Brown JR, Bharadwaj S, Becq A, Xiao X, Liu P, Li L, Song Y, Zhang D, Li Y, Xu G, Tu M, Liu X. Real-time automatic detection system increases colonoscopic polyp and adenoma detection rates: a prospective randomised controlled study. Gut. 2019;68(10):1813-1819. https://doi.org/10.1136/ gutjnl-2018-317500

18. Byrne MF, Chapados N, Soudan F, Oertel C, Linares Pérez M, Kelly R, Iqbal N, Chandelier F, Rex DK. Realtime differentiation of adenomatous and hyperplastic diminutive colorectal polyps during analysis of unaltered videos of standard colonoscopy using a deep learning model. Gut. 2019;68(1):94-100. https://doi.org/10.1136/ gutjnl-2017-314547

19. Chen PJ, Lin MC, Lai MJ, Lin JC, Lu HH, Tseng VS. Accurate Classification of Diminutive Colorectal Polyps Using Computer-Aided Analysis. Gastroenterology. 2018;154(3):568-575. https://doi.org/10.1053/j.gastro.2017.10.010

20. Wang P, Liu P, Glissen Brown JR, Berzin TM, Zhou G, Lei S, Liu X, Li L, Xiao X. Lower Adenoma Miss Rate of Computer-Aided Detection-Assisted Colonoscopy vs Routine White-Light Colonoscopy in a Prospective Tandem Study. Gastroenterology. 2020;159(4):1252-1261. e5. https://doi.org/10.1053/j.gastro.2020.06.023

21. Repici A, Badalamenti M, Maselli R, Correale L, Radaelli F, Rondonotti E, Ferrara E, Spadaccini M, Alkandari A, Fugazza A, Anderloni A, Galtieri PA, Pellegatta G, Carrara S, Di Leo M, Craviotto V, Lamonaca L, Lorenzetti R, Andrealli A, Antonelli G, Wallace M, Sharma P, Rosch T, Hassan C. Efficacy of Real-Time Computer-Aided Detection of Colorectal Neoplasia in a Randomized Trial. Gastroenterology. 2020;159(2):512-520.e7. https://doi. org/10.1053/j.gastro.2020.04.062

22. Gong D, Wu L, Zhang J, Mu G, Shen L, Liu J, Wang Z, Zhou W, An P, Huang X, Jiang X, Li Y, Wan X, Hu S, Chen 
Y, Hu X, Xu Y, Zhu X, Li S, Yao L, He X, Chen D, Huang L, Wei X, Wang X, Yu H. Detection of colorectal adenomas with a real-time computer-aided system (ENDOANGEL): a randomised controlled study. Lancet Gastroenterol Hepatol. 2020;5(4):352-361. https://doi.org/10.1016/ S2468-1253(19)30413-3

23. Hassan C, Spadaccini M, Iannone A, Maselli R, Jovani M, Chandrasekar VT, Antonelli G, Yu H, Areia M, Dinis-Ribeiro M, Bhandari P, Sharma P, Rex DK, Rösch T, Wallace M, Repici A. Performance of artificial intelligence in colonoscopy for adenoma and polyp detection: a systematic review and meta-analysis. Gastrointest
Endosc. 2021;93(1):77-85.e6. https://doi.org/10.1016/j. gie.2020.06.059

24. Aziz M, Fatima R, Dong C, Lee-Smith W, Nawras A. The impact of deep convolutional neural network-based artificial intelligence on colonoscopy outcomes: A systematic review with meta-analysis. J Gastroenterol Hepatol. 2020;35(10):1676-1683. https://doi.org/10.1111/ jgh. 15070

25. Lui TKL, Guo CG, Leung WK. Accuracy of artificial intelligence on histology prediction and detection of colorectal polyps: a systematic review and meta-analysis. Gastrointest Endosc. 2020;92(1):11-22.e6. https://doi.org/10.1016/j. gie.2020.02.033 